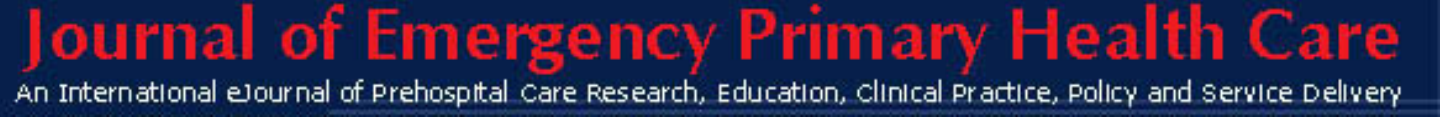

ISSN 1447-4999

\title{
WEBSITE REVIEW
}

Article 990111

\section{Emma Brandenburg}

School of Nursing and Midwifery, Griffith University

Finding websites that offer accurate and comprehensive information can have a substantial positive impact on the study and practice of primary emergency care and related fields. The Centre for Disease Control and Prevention (CDC) website at www.cdc.gov offers an extensive online resource for students and practitioners alike.

The US Department of Health and Human Services oversees the CDC, which aims to protect and promote health and safety by providing accurate information to enhance health decisions. Links and information are also available for the other agencies run by the Department of Health and Human Services, such as the Epidemiology Program office, which produces the Morbidity, Mortality Weekly Report (MMRW), and the National Centre for HIV, STD, and TB prevention.

The website is regularly evolving, providing updated information on education programs and current topics. Considering its immense size and impressive topic coverage, it is navigated easily and is simple to use, requiring only modest Internet browsing skills. The structure is logical and appealing to a wide range of users.

The CDC home page also lists health and safety topics. Of particular interest are the diseases and conditions, which are further broken down into the categories of infectious and other, disorders, chronic diseases and conditions, and risk factors. Each category links to additional topics and information. Also interesting is the information provided under the emergency preparedness and response link, which has subsections including information pertaining to mass trauma, bioterrorism, chemical emergencies, natural disasters, and recent outbreaks and incidents.

The $A-Z$ index offers an easy to use, extensive compilation of topics covered in the website, including education programs, diseases and conditions, and health promotion information. For instance, the information available in this format on Meningococcal encompasses information on the disease and health promotion data in the form of traveller's health advice. Within the Meningococcal disease link, the information is broken down into three sections: general information, technical information, and additional information. The general information covers frequently asked questions such as 'What is Meningitis?', and 'What are the signs and symptoms?' The technical information establishes information such as aetiology, clinical 
features, and sequelae. Additional information is provided in the form of links to further information, published articles, and references.

While the information available is impressive, a visual or pictorial guide to assist with recognition and diagnosis would be a valuable addition.

The CDC website serves its target audience extremely well; however, as a tool available internationally, users need to be mindful of region-specific differences in information. For example, the vaccination schedules listed are specific to the US population and vary to those used in Australia. Providing links to region-specific information or international websites could easily alleviate this.

For resources and local topic coverage, the Australian Government's Department of Health and Ageing website (located at www.health.gov.au) lists current topics, news, and issues impacting the Australian population. The information available regarding Meningococcal in the $A-Z$ index is comprehensive and, while is not as extensive as that found at the CDC website, does link to the guidelines for early clinical and public health management of Meningococcal disease in Australia.

The Australian Government's National Health and Medical Research Council website (located at www.nhmrc.gov.au) provides information and links on issues such as health advice, ethical issues, and research, and while topic specific information (such as that relating to Meningococcal) can be found, more active searching is required as there are no direct links such as an A-Z topic index.

Additionally, the HealthInsite website (located at www.healthinsite.gov.au) lists various resources and publications that provide resources at an Australian level.

Similarly, the Australian Infection Control Association's website (located at www.aica.org.au) provides information relevant to Australia, and while it does provide several important links (such as the Association for Professionals in Infection Control and Epidemiology (APIC) and Infection Control Practitioners Association of Queensland Inc (ICPAQ)), it proves to be less user friendly and does not provide the extensive topic coverage of the other sites listed above.

When actively searching for information to guide your practice, it is essential to source and critique a wide of information. The CDC website is an accurate, comprehensive, and simple to use online resource and is a valuable tool for students and practitioners; however, it may not have sufficient information specific to your needs. There are tremendous amounts of electronic literature available - the validity, reliability, and relevance of this information to your specific need or specialty are critical. Your country of origin will largely influence the applicability of electronic resources. Before going to the international literature, be sure you have thoroughly examined local, state, or national literature and resources.

Additional electronic recourses that you may find useful include: 
Queensland Health Communicable Diseases Branch

http://www.health.qld.gov.au/phs/cdu/home.asp

Health-In-Site

www.healthinsite.gov.au

National Health \& Medical Research Council www.nhmrc.gov.au

Australian Infection Control Association

www.aica.org.au

Association for Professionals in Infection Control and Epidemiology

http://www.apic.org//AM/Template.cfm?Section=Home

World Health Organisation

http://www.who.int/en/ 